\title{
Performance Comparison of Spatial Modulation Detectors Under Channel Impairments
}

\author{
Ebrahim Soujeri and Georges Kaddoum \\ University of Québec, École de Technologie Supérieure, Montréal - Canada \\ Emails: ebrahim.soujeri@lacime.etsmtl.ca, georges.kaddoum@etsmtl.ca
}

\begin{abstract}
Spatial modulation (SM) is a multiple antenna transmission approach that uses the index of the transmitting antenna for delivering information data. The choice on the transmitting antenna depends on the incoming bit sequence and has a random nature. The receiver estimates the transmitted symbol and the active transmit antenna index and uses both estimations to retrieve the original information bits. For best reception, individual channel links between transmit and receive antenna pairs must be unique. In fact, correct reception totally depends on the uniqueness of channel coefficients. Incorrectly interpreting channel state information at the receiver causes the BER performance to degrade and the detection to fall short. In this paper, we develop a channel model that incorporates both correlated and imperfect channel state information and use this model to study the bit-error-ratio (BER) performance of most widely used SM detectors, namely the minimum mean square error, the maximum likelihood and the optimum detector in flat Rayleigh fading channels to examine the sensitivity these detectors exhibit to the presence of either channel correlation or imperfect channel state information. The capacity of SM system is studied in comparison to other multiple-input multiple-output techniques and the influence of channel impairments on the overall system capacity is observed.
\end{abstract}

\section{INTRODUCTION}

Spatial Modulation (SM) is a recently developed lowcomplexity Multiple-Input Multiple-Output (MIMO) scheme where the information bitstream is divided into blocks of length $\left(n_{t}+m\right)=\log _{2}\left(N_{t} M\right)$ bits, where $N_{t}$ is the number of transmit antennae and $M$ is the size of the signal set [1, 2]. In SM, only one antenna out of $N_{t}$ antennae is active during a transmission time. In each block of $\left(n_{t}+m\right)$ bits, $m=\log _{2}(M)$ bits select a symbol from an M-ary signal set (such as M-QAM or -PSK) and $n_{t}=\log _{2}\left(N_{t}\right)$ bits select an antenna out of $N_{t}$ transmit antennae to accomplish transmission. In this configuration, transmit antenna indices will be $j=1,2,3, \ldots, N_{t}$.

The throughput achieved by this scheme is $R=\log _{2}\left(N_{t} M\right)$ bpcu. Therefore, the SM scheme achieves an increase in the spectral efficiency of $\log _{2}(M)$ bits over singleantenna systems. In order to achieve higher throughputs, either $N_{t}$ or $M$, or both need to be increased which renders this scheme suitable for low and moderately high spectral efficiencies. One of the important benefits of this scheme is that it is free from inter-channel interference (ICI) by virtue of activating a single transmit antenna at a time. In the context of the scope of this paper, the impact of imperfect

This work has been supported by the NSERC discovery grant 435243 2013. E. Soujeri is also affiliated to the Dept. of Electrical and Electronics Engineering, European University of Lefke, North Cyprus.
CSI on a multiple-input multiple-output (MIMO) system with interference has been studied in [3], the performance of a MISO system with maximum likelihood (ML) detection and full CSI at the receiver is considered in [4] and the effect of channel estimation errors on the performance of SM with ML detection is examined in [5]. Since the uniqueness of channel coefficients is a requirement for correct detection in SM system and because the system performance entirely depends on the peculiarity of channel coefficients, inaccurately depicting channel state information at the receiver leads to BER performance degradation.

In this paper, a channel model that incorporates both correlated and imperfect channel state information at the receiver is developed. The developed model is then used to study the biterror-ratio (BER) performance of three most widely used SM detectors, namely the minimum mean square error (MMSE), the $\mathrm{ML}$ and the optimum detector (OD) in flat Rayleigh fading channels to ponder the extent to which the BER performance deviates from the ideal case with perfect knowledge of channel state information (CSI). The influence of channel impairments on the capacity of the SM system under the developed model is also investigated. The rest of this paper is organized as follows: In Section II, SM system model is presented. In Section III, an explanation regarding the SM detectors on which this study focuses is presented. Section IV studies channel impairments. Capacity of SM is provided in Section V. Simulation results are presented in Section VI and discussions and conclusions are given in Section VII.

\section{SM SYSTEM MODEL}

The SM system consists of a MIMO wireless link with $N_{t}$ transmit and $N_{r}$ receive antennae, and a random sequence of independent bits $\mathbf{b}=\left[\begin{array}{llll}b_{1} & b_{2} & \cdots & b_{N_{t}}\end{array}\right]$ that enter an SM mapper or interleaver encoder, where groups of $m+n_{t}$ bits are mapped into a constellation vector $\mathbf{x}=\left[\begin{array}{llll}x_{1} & x_{2} & \cdots & x_{N_{t}}\end{array}\right]^{T}$ and are transmitted at a time. In SM, only one antenna is active during transmission and only one single entry is non-zero in $\mathbf{x}$, i.e. the entry (or index) of the chosen antenna will be nonzero. Fig. 1 gives a pictorial representation of this scheme. Note that the mapping table in Fig. 1 is for the binary transmission case and is put there for illustration simplicity.

\section{A. The Wireless Channel}

The SM signal is transmitted over an $N_{r} \times N_{t}$ wireless channel $\mathbf{H}$ and experiences an $N_{r}$-dim additive white Gaussian noise (AWGN) $\mathbf{n}=\left[\begin{array}{llll}n_{1} & n_{2} & \cdots & n_{N_{r}}\end{array}\right]^{T}$. The channel 

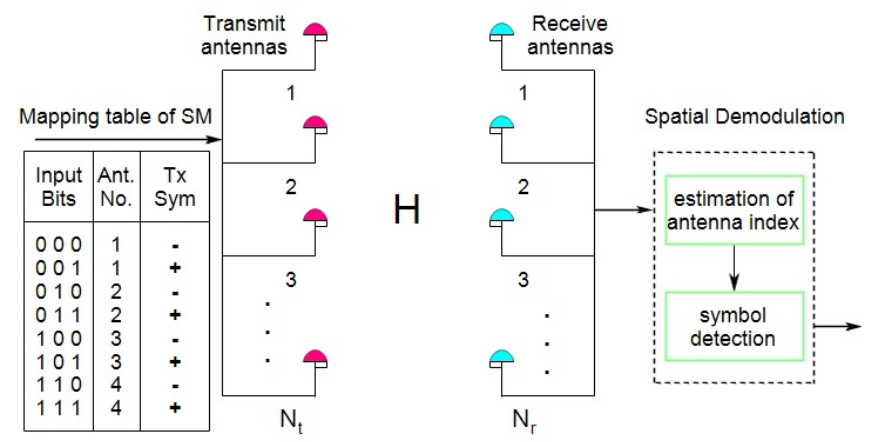

Fig. 1: The SM system.

matrix $\mathbf{H} \in \mathbb{C}^{N_{r} \times N_{t}}$ has entries given by

$$
\mathbf{H}=\left[\begin{array}{cccc}
h_{11} & h_{12} & \cdots & h_{1 N_{t}} \\
h_{21} & h_{22} & \cdots & h_{2 N_{t}} \\
\vdots & \vdots & \ddots & \vdots \\
h_{N_{r} 1} & h_{N_{r} 2} & \cdots & h_{N_{r} N_{t}}
\end{array}\right]
$$

where $h_{j i}$ is a complex fading coefficient that connects the $i^{\text {th }}$ transmit antenna to the $j^{\text {th }}$ receive antenna and is modelled as independent and identically distributed (iid) zeromean complex Gaussian (ZMCG) random variables with unit variance. Knowing that SM uses symbol indices as well as antenna indices as means to send information, the symbol index combined with the antenna index make up the SM mapper which outputs a constellation vector that has the following form

$$
\mathbf{x}_{j q}=\left[\begin{array}{ccccccc}
0 & 0 & \cdots & \begin{array}{c}
x_{q} \\
\uparrow \\
j^{t h} \text { position }
\end{array} & \cdots & 0 & 0
\end{array}\right]^{T}
$$

where $x_{q}$ is the $q^{\text {th }}$ symbol from the $M$-ary constellation $\mathcal{X}$ and $j$ is the index of the activated antenna. Therefore, only the $j^{\text {th }}$ antenna remains active transmitting the $q^{\text {th }}$ symbol. The output of the SM channel when the $q^{\text {th }}$ symbol is transmitted on the $j^{\text {th }}$ antenna is expressed as

$$
\mathbf{y}=\sqrt{E_{s}} \mathbf{h}_{j} x_{q}+\mathbf{n}
$$

where $E_{s}$ is the symbol energy defined as $E_{s} \triangleq m E_{b}, \mathbf{h}_{j}$ denotes the activation of the $j^{\text {th }}$ column of $\mathbf{H}$ for a randomly chosen $j$ during each transmission period, $\mathbf{y} \in \mathbb{C}^{N_{r} \times 1}$ is the received samples vector and $\mathbf{n} \in \mathbb{C}^{N_{r} \times 1}$ is the additive noise vector, where each element is assumed to be iid ZMCG random variable with variance $\sigma_{n}^{2}$. From this point onwards and for the sake of simplicity, we assume that the symbol energy $\sqrt{E_{s}}$ is embedded in the transmitted symbol $x_{q}$ such that the expression $\sqrt{E_{s}} x_{q}$ reduces to $x_{q}$.

\section{SM DETECTOR TyPes}

As indicated earlier, we consider the performance of the most widely used three types of SM detectors, namely the MMSE, the ML and the OD receivers. In MMSE, the antenna index is estimated first, then the transmitted symbol is detected [1]. In both ML and OD, a joint detection of antenna index and transmitted symbol is carried out. The size of the search space in MMSE is $N_{t}+M$ while it is $N_{t} M$ in ML and OD.

\section{A. Minimum Mean Square Error Detection}

The MMSE detector carries out detection at two consecutive stages; first, the antenna index is estimated, then the transmitted symbol is detected [1]. The transmitted signal is received by $N_{r}$ receive antennae. After demodulation at the $k^{\text {th }}$ antenna, the complex conjugate of the $k^{\text {th }}$ row of $\mathbf{H}$ is multiplied by $\mathbf{y}$ resulting in

$$
g_{k}=\mathbf{h}_{k}^{H} \mathbf{y}
$$

for $k=1,2, \cdots, N_{t}$. Placing the outcome for all transmit antennae coefficients in a complex vector such that

$$
\mathbf{g}=\left[\begin{array}{llll}
g_{1} & g_{2} & \cdots & g_{N_{t}}
\end{array}\right]
$$

The transmit antenna number $\hat{j}$ is the index (position) of the element in $\mathbf{g}$ whose absolute value is maximum [1]

$$
\hat{j}=\underset{j}{\arg \max }|\mathbf{g}|
$$

The antenna index (number) estimate is the position of the maximum absolute value of all elements in the vector $\mathbf{g}$. As can be noticed in the SM transmission, $\mathbf{H}$ reduces to $\mathbf{h}_{j}$ by virtue of having only one active antenna at a time, i.e. the $j^{\text {th }}$ antenna. The search argued in Eq. (6) corresponds to finding the element of greatest magnitude in the spatial domain among a set of $N_{t}$ elements. Assuming that the estimate of the transmit antenna number is correct, the transmitted symbol at this instant can be estimated by finding the distance between $g_{j}$ and each signal constellation point. That is to say, the constellation point that has minimum distance to $g_{j}$ is considered to be the winner.

$$
\hat{q}=\underset{q}{\arg \min }\left|g_{j}-x_{q}\right|
$$

\section{B. Maximum Likelihood Detection}

The ML detection which is based on maximum likelihood sequence estimation (MLSE) is a detection scheme that calculates the minimum Euclidean distance between the received signal and all possibly transmitted sequences. An example of such a detection scheme and the search for the closest lattice point for MIMO is performed in [6] and for SM-MIMO is carried out in [7]. ML estimates the combination of antenna index and transmit symbol at one shot, given by the expression

$$
[\hat{j}, \hat{q}]=\underset{j, q}{\arg \min }\left\|\mathbf{y}-\mathbf{h}_{j} x_{q}\right\|^{2}
$$

where $\|\cdot\|_{F}$ denotes the Frobenius norm which is defined as the sum of the absolute squares of its argument [8]. The ML detector jointly estimates the antenna index and the transmitted symbols by searching over all possible values of the constellation vector space $\mathcal{X}$ and channel matrix $\mathbf{H}$. It must be noted, however, that the complexity of this detector increases exponentially with the modulation order $M$ and number of transmit antennae $N_{t}$ as a natural consequence for the expansion of the search space given by $N_{t} M$.

\section{Optimal Detection}

Like the ML detector, the optimum detector (OD) also performs a joint detection of antenna indices and transmitted 
symbols, however, it follows slightly a different rule than the ML detector and is given by

$$
[\hat{j}, \hat{q}]=\underset{j, q}{\arg \min }\left(\left\|\mathbf{h}_{j}\right\|_{\mathrm{F}}^{2}\left|x_{q}\right|^{2}-2 \operatorname{Re}\left\{\mathbf{y}^{H} \mathbf{h}_{j} x_{q}\right\}\right)
$$

where the optimal detection is based on the maximum likelihood detection parameters excluding the term $\mathbf{y}^{H} \mathbf{y}$.

\section{CHANNEL IMPAIRMENTS}

The channel impairments we attempt to study in this work are classified in two categories. First we study the effect of channel estimation errors (imperfections) on the performance of SM detectors, then we investigate the influence of channel correlation. The former is the case in which the estimated channel coefficients are contaminated with noise. The latter is the case where channel coefficients are correlated.

1) Erroneous Channel: Channel estimation is the most crucial and important part of SM detection process. We assume the presence of either error-free (perfect) or erroneous (imperfect) channel estimations. Since avoiding CSI errors is difficult in practical systems, we assume the existence of both amplitude and phase errors in the estimated CSI. We define erroneous CSI such that the following relationship between the estimated and the correct channel coefficients at the $k^{\text {th }}$ receiver holds

$$
\hat{\mathbf{h}}_{k}=\rho \mathbf{h}_{k}+(1-\rho) \varepsilon^{1 \times N_{t}}
$$

where $\varepsilon$ is a normal distributed random variable with zero mean and unit variance. The coefficient $0<\rho<1$ is a correspondence factor that determines the resemblance of the estimated CSI to the actual one. Perfect CSI estimation is achieved when $\rho=1$, i.e. when $1-\rho=0$.

2) Correlated Channel: In the context of our work, we define channel correlation to be the interference of the entries of the channel matrix with each other such that the correlated channel becomes $\widehat{\mathbf{H}}=\mathbf{H} \Delta$, where $\Delta \in \mathbb{R}^{N_{t} \times N_{t}}$ is a Toepltiz matrix with the value $1-\delta$ in the diagonal elements and the value $\delta$ in the superdiagonal and subdiagonal elements. The $\Delta$ matrix takes the form given below for $N_{t}=4$

$$
\Delta=\left[\begin{array}{cccc}
(1-\delta) & \delta & 0 & 0 \\
\delta & (1-\delta) & \delta & 0 \\
0 & \delta & (1-\delta) & \delta \\
0 & 0 & \delta & (1-\delta)
\end{array}\right]
$$

where $0<\delta \ll 1$. Channel coefficients are said to be perfectly uncorrelated when $\delta=0$, i.e. when $1-\delta=1$.

3) Erroneous and Correlated Channel: We consider the generalized case where the channel coefficients are first correlated with each other, then undergo certain amount of contamination with noise. We have used the following model to incorporate both correlation and contamination with noise

$$
\widetilde{\mathbf{H}}=\rho \mathbf{H} \Delta+(1-\rho) \varepsilon^{N_{r} \times N_{t}} .
$$

It is clear that the overall channel model $\widetilde{\mathbf{H}}$ depicted in Eq. (12) is governed by the two parameters $\delta$ and $\rho$ each with the properties described aforetime and is the the result of having $\mathbf{H}$ be first correlated by $\Delta$ in the fashion expressed in Eq. (11) and then contaminated by noise in a similar manner to Eq. (10).

\section{CAPACITY OF SM}

The capacity of MIMO, single-input multiple-output (SIMO) and multiple-input single-output (MISO) systems are well discussed in Chapter 8 of [9] where the capacities of these systems are compared and graphically presented for $n=1,2, \cdots, 16$ for the $n \times n$ MIMO, $n \times 1$ SIMO and $1 \times n$ MISO cases where $n=N_{t}=N_{r}$. In this work, we reproduce the capacities described and shown in Fig. 8.6 on page 345 of [9] and we base our argument on capacity by adding the capacity of SM to this collection in order to have a better outlook on the location of SM, in terms of capacity, among the other MIMO technologies mentioned here.

\section{A. Capacity Calculation}

In order to calculate the capacity of the SM system, the MIMO capacity approach will not apply because the antenna number represents added information. Furthermore, the antenna pattern is considered as spatial constellation and not as source of information. Thus, concerning computing the SM capacity, the conventional information theory approach will be used [10]

$$
C_{S M}=\left(m+n_{t}\right)\left[1+p_{e} \log _{2}\left(p_{e}\right)+\left(1-p_{e}\right) \log _{2}\left(1-p_{e}\right)\right]
$$

where $p_{e}$ is the probability of error of SM detection at a given SNR value and $m+n_{t}$ represents the total number of bits conveyed by the SM system. Since $N_{t}$ has to be a power of 2 in SM systems, the number of antennae $n$ must be set to $2,4,8$ or 16 and the SM capacity must be calculated based on the probability of error for the $2 \times 2,4 \times 4,8 \times 8$, and $16 \times 16$ configurations which correspond to number of antenna indexing bits $n_{t}$ to be $1,2,3$ and 4 respectively. Note that the configuration size follows from $n=N_{t}=N_{r}$ as indicated earlier. A flashback on the capacity issue along with the obtained capacity results are presented in Section VI.

\section{Simulation Results}

In this section we provide simulation results for the SM scheme in Rayleigh fading channels using Monte Carlo simulation for the MMSE, ML and Optimum detectors. Various values for modulation order $M$ has been used but the antenna configuration has been restricted to $4 \times 1$ and $4 \times 4$. The case of perfect and imperfect CSI has been studied first, then the effect of channel correlation is investigated. The destructive effects of CSI imperfection and correlation on the BER performance and on the capacity of SM systems is explored. The partially estimated CSI case comprises two situations, the first is when the CSI estimation is erroneous (imperfect) and is contaminated with noise, the second is when channel coefficients are correlated CSI, i.e. the coefficients do interfere among themselves. In this work, we study the consequences of erroneous and correlated CSI on the BER performance and on the capacity of SM system. The noise at each antenna is assumed to be independent zero-mean complex Gaussian random variable with variance $\sigma$.

\section{A. The Perfect CSI case}

To have a clear vision on the performance of SM detectors presented in this paper, we first have a look at the perfect CSI case where channel coefficients are perfectly estimated. Fig. 


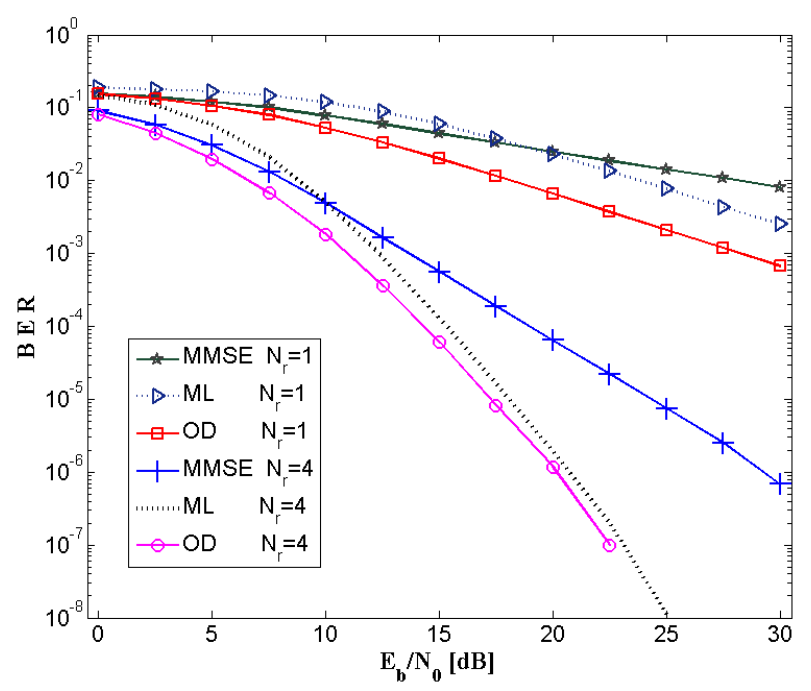

Fig. 2: BER performance of SM with perfectly estimated CSI for $4 \times 1$ and $4 \times 4$ configurations.

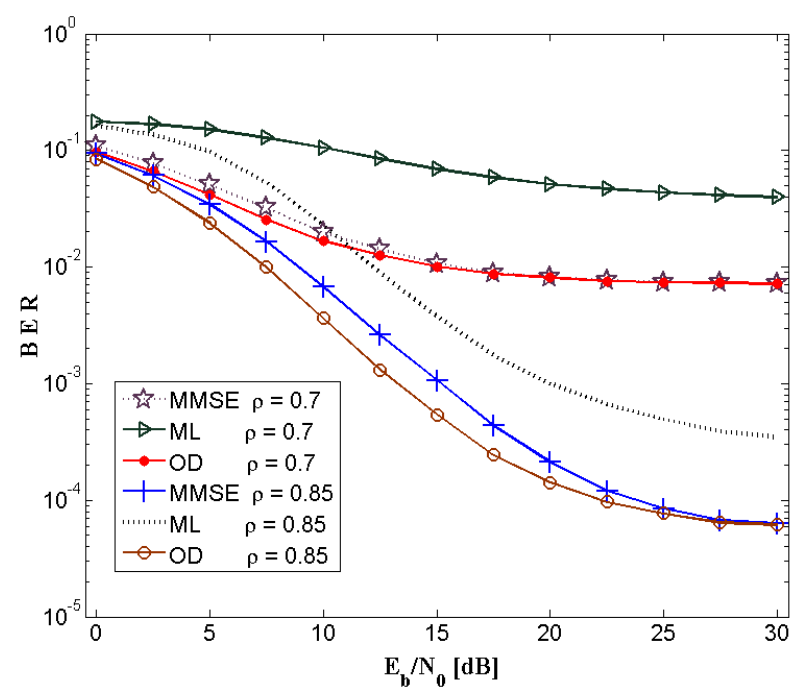

Fig. 3: BER performance of SM with imperfect CSI for a $4 \times 4$ configuration with $M=8$.

2 demonstrates the BER performance of the system for the $4 \times 1$ and $4 \times 4$ cases, both with a modulation order $M=8$. As expected and observed in Fig. 2, the optimum detector shows superior performance in comparison to its rivals in this scenario and antenna diversity plays its BER enhancement role as expected.

\section{B. The Imperfect CSI case}

In this section, we study the performance of SM detectors with imperfect channel coefficients as narrated in Eq. (10). The BER performance of SM detectors for $\rho=0.85$ and $\rho=$ 0.7 is shown in Fig. 3. As obsreved, the BER performance deteriorates with decreased value of the correspondence factor $\rho$. The ML detector seems to be more susceptible to noise since it contains more noisy terms that challenge the minimum Euclidean distance.

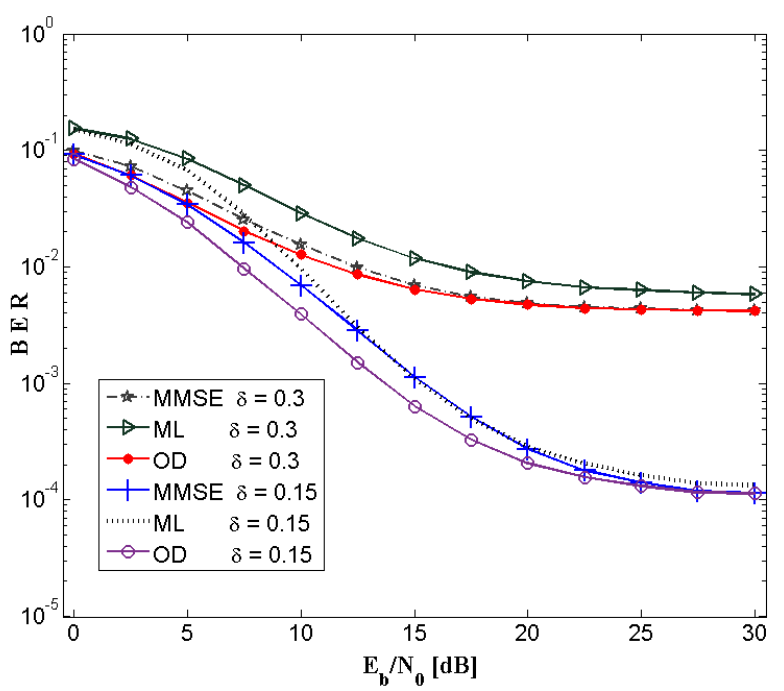

Fig. 4: BER performance of SM with correlated CSI for a $4 \times 4$ configuration with $M=8$.

\section{The Correlated CSI case}

The BER performance of the SM system with correlated channel coefficients as described by the $\Delta$ matrix in Eq. (11) is considered here. The performance of SM detectors for $\delta=$ 0.15 and $\delta=0.3$ is shown in Fig. 4. As can be observed in this figure, the BER performance deteriorates as the correlation value increases.

\section{Correlated and Imperfect CSI}

The system has been tested in accordance with the inclusive model we propose in Eq. (12) with $M=8, N_{t}=N_{r}=4$. A 3-D view of the BER using OD SM versus $\delta$ and $1-\rho$ is shown in Fig. 5 for $0 \leq \delta \leq 0.5$ and $0 \leq 1-\rho \leq 0.5$. The reason why we chose the optimum detector is because this detector proved to outperform the rival detectors while it maintained more robustness against both imperfection and correlation. As observed here, both factors $\rho$ and $\delta$ exhibit a similar destructive trend. The relationship between $\rho, \delta$ and the noise variance is left for future analysis.

\section{E. Capacity Issues}

Having studied the influence of imperfect and correlated CSI on the system performance, we will have a look at the capacity degradation under the presence of $\rho$ and $\delta$. Based on our results, the OD has proved to achieve the best BER performance while it exhibits most robustness against channel imperfections and correlation, so the probability of error in Eq. (13) will be based on optimal detection. Furthermore, the signal to noise ratio will be fixed at $0 \mathrm{~dB}$ since the MIMO, MISO and SIMO systems of [10] are evaluated at this value. In Fig. 6 the capacities originally given in [9] were reproduced and the capacity of SM was then calculated and added in the described fashion. Since MIMO, SIMO and MISO evaluations are done at perfect CSI, i.e. $\rho=1, \delta=0$, the capacity of SM at these values corresponds to the perfect CSI case and represents the capacity of SM in comparison with other MIMO technologies. As seen in Fig. 6, with imperfect and correlated 


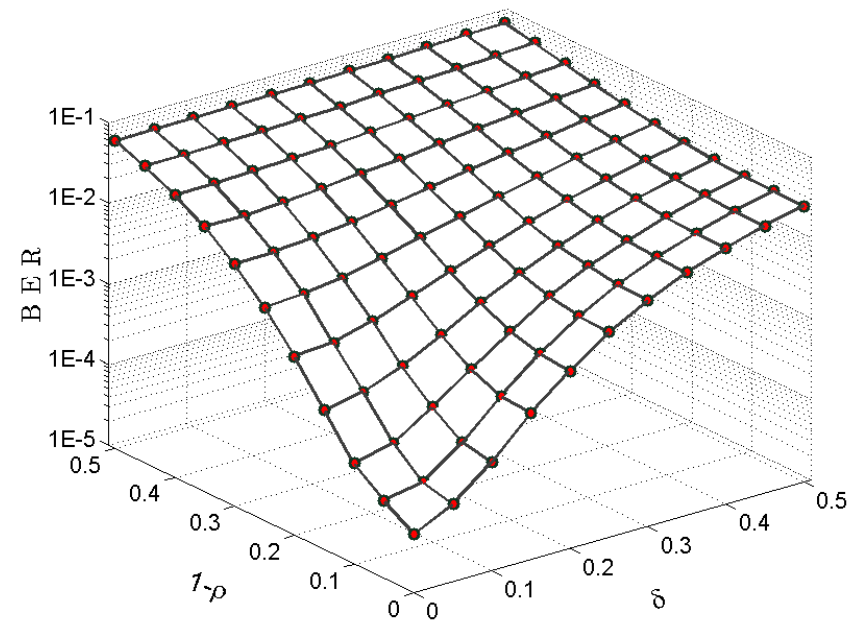

Fig. 5: BER performance of SM vs. correlated and imperfect CSI estimation at $E_{b} / N_{0}=15 \mathrm{~dB}$.

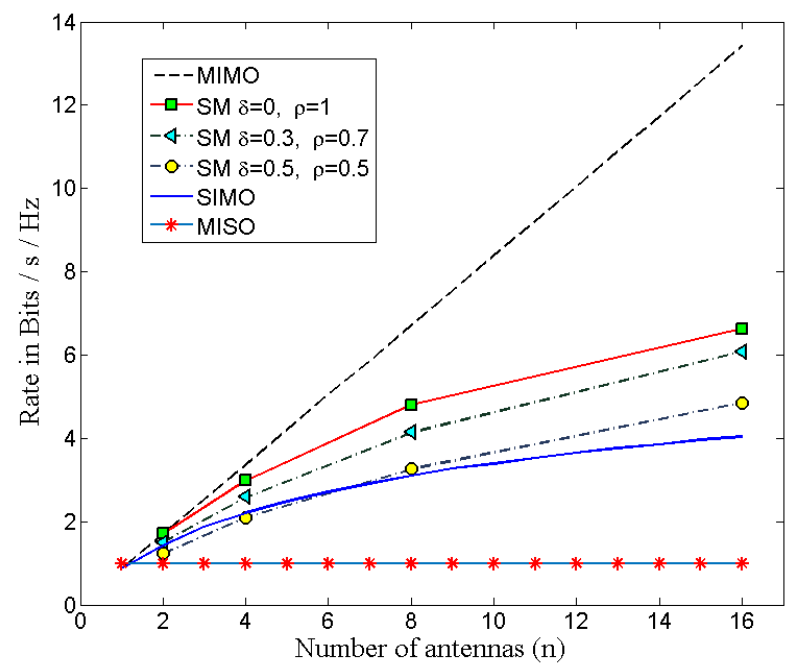

Fig. 6: Capacities of MIMO, SM, SIMO and MISO channels

coefficients, capacity of the SM system drops as a consequence of the BER performance decline at no surprise. The capacity of SM deteriorates even more as $\rho$ and $\delta$ take farther distances from the ideal values. More on MIMO capacity issues may be looked up in [11] and [12].

\section{Discussions AND CONCLUSIONS}

In this work, we have developed a general model for channel impairments that incorporates channel imperfection and channel correlation. The developed model is then used to study the effects of channel impairments on the BER performance of three most widely used SM receivers, namely the MMSE, ML and OD receivers. Moreover, the influence of channel impairments on the capacity of the SM system has also been investigated. Even though numerical calculations are mainly based on a $4 \times 4$ SM system configuration, the results obtained may be generalized to any accommodating configuration. Furthermore, our results reveal that the BER performance of SM detectors under study deteriorate with channel imperfections especially in high SNR regions. In fact, the performance of the ML is very fragile and sensitive to either correlated or imperfect channel coefficients while MMSE and OD do better in such situations. Given the lower complexity of MMSE compared to OD and the convergence of their BER performance at high SNR values, the MMSE might be a preferred candidate for higher power scenarios where simplicity matters. However, at lower SNR, the OD becomes a more suitable choice. Fundamental complexity concepts are provided in [13] while more related complexity discussions are found in $[1,2,14]$ and is not repeated here. The information theory-based calculation of the SM capacity carried out in this work confirms and approves this fact. Consequently, channel impairments cause the SM system capacity to drop as a reasonable and logical aftermath of the rise in the probability of error. Therefore, CSI is a phenomenon that can not be always assumed to be perfect because of the serious impacts it has on the BER performance and on the capacity of the SM system. Finally, we observe that SM is sensitive to both imperfect and correlated channels.

\section{REFERENCES}

[1] R. Mesleh, H. Haas, C. W. Ahn, and S. Yun, "Spatial modulationa new low complexity spectral efficiency enhancing technique," in Communications and Networking in China, 2006. ChinaCom'06. First International Conference on. IEEE, 2006, pp. 1-5.

[2] R. Y. Mesleh, H. Haas, S. Sinanovic, C. W. Ahn, and S. Yun, "Spatial modulation," Vehicular Technology, IEEE Transactions on, vol. 57, no. 4, pp. 2228-2241, 2008.

[3] N. Lee, O. Simeone, and J. Kang, "The effect of imperfect channel knowledge on a mimo system with interference," IEEE Transactions on Communications, vol. 60, no. 8, pp. 2221-2229, 2012.

[4] M. Di Renzo and H. Haas, "A general framework for performance analysis of space shift keying (ssk) modulation for miso correlated nakagami-m fading channels," Communications, IEEE Transactions on, vol. 58, no. 9, pp. 2590-2603, 2010.

[5] R. Mesleh and S. S. Ikki, "On the effect of gaussian imperfect channel estimations on the performance of space modulation techniques," in Vehicular Technology Conference (VTC Spring), 2012 IEEE 75th. IEEE, 2012, pp. 1-5.

[6] M. O. Damen, H. El Gamal, and G. Caire, "On maximum-likelihood detection and the search for the closest lattice point," Information Theory, IEEE Transactions on, vol. 49, no. 10, pp. 2389-2402, 2003.

[7] R. M. Legnain, R. H. Hafez, I. D. Marsland, and A. M. Legnain, "A novel spatial modulation using mimo spatial multiplexing," in Communications, Signal Processing, and their Applications (ICCSPA), 2013 1st International Conference on. IEEE, 2013, pp. 1-4.

[8] G. H. Golub and C. F. Van Loan, Matrix computations. JHU Press, 2012, vol. 3 .

[9] T. David and V. Pramod, "Fundamentals of wireless communication," New York, Cambridge, 2005.

[10] F. A. Prisecaru, "Mutual information and capacity of spatial modulation systems."

[11] G. J. Foschini, "Layered space-time architecture for wireless communication in a fading environment when using multi-element antennas," Bell labs technical journal, vol. 1, no. 2, pp. 41-59, 1996.

[12] A. Kalis, A. G. Kanatas, and C. B. Papadias, "A novel approach to mimo transmission using a single rf front end," Selected Areas in Communications, IEEE Journal on, vol. 26, no. 6, pp. 972-980, 2008.

[13] L. Bai, J. Choi, and Q. Yu, Low Complexity MIMO Receivers. Springer, 2014.

[14] G. Kaddoum and E. Soujeri, "On the comparison between code-index modulation and spatial modulation techniques," in The International Conference on Information and Communication Technology Research UAE, 2015. ICTRC'15. IEEE, 2015, pp. 1-4. 